\title{
Twitter and Academic Urology in the United States and Canada: A Comprehensive Assessment of the Twitter-verse in 2019.
}

\author{
Thenappan Chandrasekar \\ Thomas Jefferson University \\ Hanan Goldberg \\ State University of New York Upstate Medical University \\ Zachary Klaassen \\ Augusta University - Medical College of Georgia; Georgia Cancer Center \\ Christopher J. Wallis \\ University of Toronto; anderbilt University Medical Center \\ f8pow Ytapts Lefangetditional works at: https://jdc.jefferson.edu/urologyfp \\ Thomas Jefferson University \\ Part of the Social Media Commons, and the Urology Commons

\section{Let us know how access to this document benefits you}

\section{Recommended Citation}

Chandrasekar, Thenappan; Goldberg, Hanan; Klaassen, Zachary; Wallis, Christopher J.; Leong, Joon Yau; Liem, Spencer; Teplitsky, Seth; Noorani, Rodrigo; and Loeb, Stacy, "Twitter and Academic Urology in the United States and Canada: A Comprehensive Assessment of the Twitter-verse in 2019." (2019). Department of Urology Faculty Papers. Paper 53.

https://jdc.jefferson.edu/urologyfp/53

This Article is brought to you for free and open access by the Jefferson Digital Commons. The Jefferson Digital Commons is a service of Thomas Jefferson University's Center for Teaching and Learning (CTL). The Commons is a showcase for Jefferson books and journals, peer-reviewed scholarly publications, unique historical collections from the University archives, and teaching tools. The Jefferson Digital Commons allows researchers and interested readers anywhere in the world to learn about and keep up to date with Jefferson scholarship. This article has been accepted for inclusion in Department of Urology Faculty Papers by an authorized administrator of the Jefferson Digital Commons. For more information, please contact: JeffersonDigitalCommons@jefferson.edu. 


\section{Authors}

Thenappan Chandrasekar, Hanan Goldberg, Zachary Klaassen, Christopher J. Wallis, Joon Yau Leong, Spencer Liem, Seth Teplitsky, Rodrigo Noorani, and Stacy Loeb 
Title: Twitter and Academic Urology in the United States and Canada: A Comprehensive Assessment of the

Twitter-verse in 2019.

Authors: Thenappan Chandrasekar,1 Hanan Goldberg,2 Zachary Klaassen,3 Christopher JD Wallis,4Joon Yau Leong,1 Spencer Liem,1 Seth Teplitsky,1 Rodrigo Noorani,5 Stacy Loeb6

Affiliation:

1. Department of Urology, Sidney Kimmel Cancer Center, Thomas Jefferson University, Philadelphia PA, USA

2. Department of Urology, State University of New York Upstate Medical University, Syracuse, NY, USA.

3. Division of Urology, Department of Surgery, Augusta University - Medical College of Georgia, Augusta, GA; Georgia Cancer Center, Augusta, GA

4. Division of Urology, Department of Surgery, University of Toronto, Toronto, ON, Canada; Department of Urology, Vanderbilt University Medical Center, Nashville, TN, USA

5. Division of Urology, Department of Surgery, University of Toronto, Toronto, Ontario, Canada

6. Department of Urology and Population Health, New York University and Manhattan Veterans Affairs, New York, NY, USA

\section{ABSTRACT}

Objective: To provide the first comprehensive analysis of the Twitter-verse amongst academic urologists and programs in North America.

\section{Methods:}

Using national accreditation and individual program websites, all active urology residency programs (USA \&

Canada) and academic Urology faculty at these programs were identified. Demographic data for each program

(AUA section, resident class size) and physician (title, fellowship training, Scopus $\mathrm{H}$-index and citations) were documented. Twitter metrics (Twitter handle, date joined, \# tweets, \# followers, \# following, likes) for programs and physicians were catalogued (data capture: March-April 2019). Descriptive analyses and temporal trends in Twitter utilization amongst programs and physician were assessed. Multivariable (MV) logistic regression was used to identify predictors of Twitter use. 
RESULTS

156 academic programs (143 USA, 13 Canada) and 2214 academic faculty (2015 USA, 199 Canada) were identified. Twitter utilization is currently $49.3 \%$ and $34.1 \%$ amongst programs and physicians, respectively, and continues to increase. On MV analysis, programs with 3-5 residents/year and programs with a higher percentage of faculty Twitter engagement were more likely to have Twitter accounts. From a physician perspective, those with fellowship training, lower academic rank (clinical instructor, assistant professor, associate professor vs. professor) and higher $\mathrm{H}$-indices were more likely to have individual Twitter accounts.

\section{CONCLUSION}

There is a steady increase in Twitter engagement amongst Urology programs and academic physicians. Faculty Twitter utilization is an important driver of program Twitter engagement. Twitter SoMe activity is strongly associated with academic productivity, and may in fact drive academic metrics. Within Urology, SoMe presence appears to be proportional to academic activity.

Keywords: Twitter, Urology, Academics, Social Media, Survey, Kardashian Index 
INTRODUCTION

Social Media ("SoMe") encompasses a broad spectrum of public use platforms, including Twitter, YouTube and Facebook that have changed the way people interact and communicate. Its integration into the medical community has been no exception. ${ }^{1,2}$ Yet, amongst the various SoMe platforms, Twitter has gained an impressive foothold within the medical community. Twitter is unique from the other platforms in that it limits posts to 280 characters (previously 140 characters) or less, emphasizing brevity. In doing so, it has provided an opportunity for rapid dissemination of medical knowledge and content amongst medical professionals and to the general public. ${ }^{1,3-5}$

Within academic medicine, Twitter has become integrated into the fabric of academic discussion and knowledge dissemination. Its role in highlighting key developments at medical conferences in real-time, using established conference hashtags, has been previously reported. ${ }^{5}$ Many physicians report using this as an important method to stay informed regarding medical advancements in their field. ${ }^{5-7}$ Its ability to facilitate international academic discussion has subsequently led to SoMe journal clubs and crowdsourcing of medical opinion. ${ }^{1,5,8}$ Medical journals have also been actively courting Twitter users, by having journal specific Twitter accounts and publishing SoMe friendly versions of manuscripts. ${ }^{6,9-11}$

Urologists have long been early adopters of new technology and SoMe is no exception. ${ }^{12-14}$ Within academic Urology, Twitter has been an important mediator of national and international conferences, ${ }^{7,15,16}$ while also serving as a platform for Urology-specific journal clubs (\#urojc, \#prostatejc, \#pedurojc) and crowdsourcing about clinical cases. ${ }^{17-19}$ This has led to both international guidelines and standardized ontology. ${ }^{20-22}$ Yet, more recently, it has become evident that Twitter is a platform for self-promotion - for Urology programs and individual physicians. ${ }^{23}$ Individual departments have utilized Twitter to highlight work by staff and trainees, 
while individual physicians utilize Twitter to shine a spotlight on academic output, which may be associated with increased citations and academic recognition. ${ }^{23,24}$

Despite this, little is known about the current landscape of Twitter utilization amongst academic Urology programs and physicians. In this study, we aim to provide the first comprehensive analysis of the Twitter-verse amongst academic urologists and programs in North America. 


\section{METHODS}

\section{Population and Demographics}

Using the Accreditation Council for Graduate Medical Education (ACGME), ${ }^{25}$ the Canadian Resident Matching Service $(\mathrm{CaRMS})^{26}$ and individual program websites, all active accredited urology residency programs in the United States and Canada were identified. For each program, state (US programs), American Urologic Association (AUA) section, and resident class size were recorded.

Academic faculty at all programs (excluding part-time faculty, clinical fellows, non-Urologists, and research faculty) were then identified and catalogued (data capture: March-April 2019) by cross-referencing all available institutional and hospital data. For each individual physician, gender, academic title, fellowship training and type(s), and AUA section were documented. Academic productivity was characterized by $\mathrm{H}$-index and citations, per Scopus records (April-May 2019), and stratified by quartiles.

\section{$\underline{\text { Twitter Metrics }}$}

Comprehensive searches were completed for all programs and physicians (April-May 2019) for the following data: Twitter handle, date joined (MM/YY), number of tweets, number of followers, number following, and number of "likes". Program Twitter accounts were limited to those specifically for the Urology Department or Division. Physician Twitter accounts were included regardless of personal, professional or mixed use. For physicians with a Twitter account, the Kardashian index (K-Index), previously described by Hall et al.., ${ }^{27}$ was calculated. The number of Twitter followers is plotted against the number of citations, serving as a surrogate measure of SoMe activity relative to academic productivity. Then, based on linear regression, the expected number of followers was calculated for each physician. The K-index is defined as the ratio of actual to expected number of followers, and was stratified into low $(<=0.25)$, expected (0.26-5.0) and high $(>5.0) .{ }^{27}$ In a 
separate analysis, we further stratified physicians into 4 quadrants based on median number of followers and citations for the entire cohort.

\section{$\underline{\text { Statistical Analysis }}$}

Descriptive statistics for demographic and Twitter utilization comparisons were performed using analysis of variance (ANOVA) for continuous variables and chi-square test for categorical variables. Temporal trends in Twitter utilization amongst programs and physicians were evaluated. Multivariable logistic regression was completed to identify predictors of Twitter use amongst physicians and programs. All statistical tests were two-tailed and a p-value of $<0.05$ was considered statistically significant. Analyses were completed using SPSS $^{\circledR}$, version 23.0 . 


\section{RESULTS}

\section{Demographics}

Through the ACGME, CaRMS, and individual programs' websites, 156 academic programs were identified (143 USA, 13 Canada). Demographics of the programs, stratified by Twitter utilization, are summarized in Table 1. 77 programs (49.3\%) had program Twitter accounts as of May 2019. Programs in the United States $(p=0.047)$, programs with more residents per year $(p=0.007)$, and programs with a higher percentage of faculty with individual Twitter accounts $(p<0.001)$ were more likely to have accounts.

Using program data, 2214 academic urology faculty (2015 USA, 199 Canada) were identified. Of these, 756 physicians (34.1\%) had a Twitter account as of May 2019. Table 2 highlights the demographics of all physicians, stratified by Twitter utilization. Fellowship trained physicians, particularly those in Urologic Oncology, Minimally Invasive Urology, and Endourology, are more likely to have a Twitter account $(p<0.001)$. Physicians with higher $\mathrm{H}$-indices $(\mathrm{p}<0.001)$ and greater number of citations $(\mathrm{p}<0.001)$ are also more likely to have a Twitter account. AUA section was also associated with Twitter account utilization ( $p=0.049)$.

\section{Twitter Account Uptake Trends over time}

Temporal trends in Twitter account utilization are highlighted in Figure 1, with Figure $1 \mathrm{~A}$ and $1 \mathrm{~B}$ focusing on programs and physicians, respectively. The first program account was created in April 2009, and as of May $2019,49.3 \%$ of programs had Twitter accounts. The first physician account was created in January 2007, and as of May 2019, 34.1\% of physicians had a Twitter account. There is a steady rise in utilization amongst both physicians and programs.

\section{$\underline{\text { Twitter Metrics }}$}


Supplementary Table 1 and 2 summarize the Twitter activity metrics for academic programs and individual physicians, respectively. With respect to programs, an increasing resident class size and increasing percentage of faculty with individual accounts was associated with a greater number of followers for the program's twitter account $(p<0.05)$. Additionally, an increasing percentage of faculty with individual accounts was associated with increased number of tweets by the program account $(p<0.05)$. Looking at physicians with Twitter accounts, female physicians have had open accounts for significantly less time than male physicians (43.00 months vs. 62.95 months, $p<0.05$ ). Fellowship training was associated with a higher number of published tweets (96.50 vs. 62.00, $p<0.05$ ). Further, there was a significant association between physicians' $\mathrm{H}$-index and number of followers; however, these individuals were less likely to "like" others' tweets. Similarly, physicians with higher number of citations were associated with more mature twitter accounts, a greater number of tweets, have a greater number of followers, but were less likely to "like" others' tweets.

\section{Predictors of Having Twitter Account}

Predictors of Twitter account utilization by programs (Table 3) and physicians (Table 4) were also assessed. Programs with 3-5 residents per year (vs. 1 resident: 4-5 [OR 11.4, 95Cl 1.67-77.83, $\mathrm{p}=0.01], 3$ [OR 6.72, 95CI 1.54-29.31, $p=0.01]$ ) and programs with a higher percentage of faculty with individual Twitter accounts (vs. 025\%: $26-50 \%$ [OR 9.06, 95Cl 3.49-23.52, $\mathrm{p}<0.01], 51-75 \%[28.39,95 \mathrm{Cl} 6.02-133.91, \mathrm{p}<0.01])$ were the most likely to have accounts. Physicians who were fellowship trained (vs. not fellowship trained: [OR 2.00, 95Cl 1.54-2.56, $\mathrm{p}<0.01]$ ), physicians of lower academic rank (vs. professors: associate professors [OR $1.38,95 \mathrm{Cl}$ 1.03-1.86, $p=0.03$ ], assistant professors [OR 2.57, $95 \mathrm{Cl} 1.64-3.07, \mathrm{p}<0.01$ ], clinical instructors [OR $2.00,95 \mathrm{Cl}$ 1.01-3.92, p 0.04]) and physicians with higher $\mathrm{H}$-indices (vs. quartile 1: quartile 2 [OR 1.70, 95Cl 1.07-2.70, p= 
0.03], quartile 3 [OR 2.57, 95CI 1.40-4.70, p < 0.01]) were more likely to have an individual Twitter account. There was no association with country, gender or number of citations with individual account utilization.

\section{Correlation with Academic Productivity}

In Figure 2, we correlate SoMe activity and academic productivity. Figure 2A focused on the K-index for all academic urologists with a Twitter account, as described by Hall et al. Of 752 physicians with Twitter accounts, 23 (3.1\%) had a high K-index. Of note, an analysis based on gender identified proportionate representation in all three categories. Figure 2B stratifies all physicians into four quadrants based median number of followers (191.5) and median number of citations (764.5) for the entire cohort. 


\section{DISCUSSION}

The utilization of SoMe within academic medicine is undoubtedly increasing and, at this time, Twitter appears to be a leading platform. In this study, we provide the first comprehensive analysis of Twittersphere amongst academic Urology programs and physicians in the United States and Canada.

In a broad assessment of all 2214 urologists at academic Urology programs, as of May 2019, Twitter utilization was at $34.1 \%$ - but rising steadily over the past ten years (Figure 1B). On multivariable analysis, fellowship trained physicians were twice as likely to have a twitter account, and as seen by Twitter metrics, were more likely to publish tweets actively rather than passively interact. Furthermore, physicians of lower academic rank (clinical instructors, assistant professors and associate professors) were more likely to utilize Twitter accounts. While age was not captured in this dataset, academic title may serve as a surrogate for physician age, and reflect adoption in a younger physician cohort. Importantly, gender was not associated with Twitter utilization. Overall, these trends indicate that Twitter utilization is on the rise amongst urologists, particularly in fellowship trained physicians who are earlier in their academic careers.

As an increasing number of urologists undergo fellowship training prior to taking an academic position, the make-up of academic programs is rapidly changing. When looking at the 156 academic programs in the US and Canada, 49.3\% had program specific Twitter accounts - and this too has been rising steadily (Figure $1 \mathrm{~A})$. In 2017, Farber et al., in a smaller survey of programs only, noted a 30\% Twitter utilization amongst Urology programs at the time of publication. ${ }^{28}$ We found that residency class size was also an independent predictor of having a Twitter account. This is intuitive, as larger residency programs are typically at academic institutions with higher volume, greater funding, and larger number of faculty. However, it should be noted that that the strongest independent predictor of a program having a Twitter account is the percentage of its academic 
faculty that are on Twitter themselves. Inherently, this implies that SoMe active faculty help drive their programs' SoMe activity. As more faculty become SoMe and Twitter savvy, we can likely expect a greater number of programs to develop a SoMe presence. Indeed, an increasing percentage of faculty with individual accounts was associated with increased number of tweets by the program account, indicating higher active engagement by the program.

At a physician level, there was an important association between academic productivity and Twitter utilization and metrics. Physicians with higher $\mathrm{H}$-indices and citations were associated a higher number of followers, indicating that academic productivity may drive SoMe following. However, those same physicians were less likely to engage other followers with "likes," suggesting one-way engagement and dissemination of thought. Importantly, on multivariable analysis, physicians with higher $\mathrm{H}$-indices (vs. quartile 1: quartile 2 [1.70, $\mathrm{p}=$ $0.03]$, quartile 3 [2.57, $p<0.01]$ ) were more likely to have an individual Twitter account, though number of citations did not have the same association. Prior studies have demonstrated an important impact of SoMe activity on increasing recognition of academic contributions. Hayon et al. recently noted that manuscripts mentioned on Twitter had a 2-fold increase in citations, while author-tweeted articles were associated with a 12.3 (2.0-fold) mean citation increase in Scopus. ${ }^{23}$ Similarly, in a broader analysis of 4208 tweets citing 286 distinct articles, Eysenbach et al. noted a highly statistically significant association between citation status and Twitter activity for a given article, and indeed, top-tweeted articles predicted top-cited articles with $93 \%$ specificity and $75 \%$ sensitivity. ${ }^{6}$ Ultimately, while causal relationships cannot be established, clearly academic productivity and recognition are linked with SoMe activity.

First described by Hall et al., ${ }^{27}$ the Kardashian index stems from the concept that some individuals are "famous for being famous," rather than their contributions to the published literature. Especially in the era of SoMe, 
with rapid dissemination of thoughts, it is feasible to have a high-profile career without comparable scientific contribution. However, it is important to note that only 23 (3.1\%) of all academic Urologists with Twitter accounts met Hall's criteria for being a 'Science Kardashian', which was arbitrarily defined as an observed-toexpected follower ratio of $>5.0$ and has never been tested in urology (Figure 2A). It would seem, at least within Urology, SoMe following correlates with academic productivity. In Figure 2B, we note that physicians in the upper left quadrant (low citations, high followers) represent those with greater SoMe following than academic output, while those in the lower right quadrant (high citations, low followers) represent physicians who are under-recognized on SoMe for their academic contributions. Most of the 23 'Science Kardashians' (based on Hall's threshold) are in the upper right quadrant (high followers, high citations), and perhaps warrant their SoMe following due to their greater than average academic productivity. Most importantly, however, we feel that this graphical representation helps highlight the individuals who are unrecognized on SoMe for their academic contributions - and the lost value of their potential impact on the SoMe environment. Spread of misinformation on social media about urological conditions is an increasingly recognized problem, ${ }^{29}$ highlighting the importance of active participation from key opinion leaders to disseminate high-quality information.

Our study is not devoid of limitations. First, as the capture of all programs' and physicians' Twitter accounts and metrics and Scopus academic metrics were manual, individual accounts may have been missed. In Scopus, many physicians had multiple listings due to prior academic affiliations, which were collated when identifiable. Ultimately, however, as an extensive effort to identify all Twitter accounts through multiple methods (broad Google and Twitter based searches using multiple key words and name combinations, search of affiliated Twitter accounts), and as most physicians were identified on Scopus, the authors are confident that the capture rate was quite high. Second, as Twitter engagement continues to change, many metrics may change 
with time. Third, Twitter accounts were captured regardless of personal or professional use, so metrics may not always reflect professional engagement. However, this study still represents the first cross-sectional assessment of academic program and physician Twitter use in the U.S. and Canada. Finally, this data is confined to North American Urology programs and remains to be assessed amongst academic programs in other parts of the world.

\section{CONCLUSION}

In this comprehensive analysis of the Urology Twitterverse, we demonstrate a steady increase in Twitter engagement amongst Urology programs and academic physicians. Faculty Twitter utilization appears to be an important driver of program Twitter engagement. Twitter SoMe activity is strongly associated with academic productivity, and may in fact drive academic metrics - but, within Urology, SoMe presence appears to be proportional to academic activity.

ACKNOWLEDGEMENTS: None 


\section{REFERENCES / BIBLIOGRAPHY}

1. Pershad Y, Hangge PT, Albadawi H, Oklu R. Social medicine: Twitter in healthcare. J Clin Med. 2018;7(6):10.3390/jcm7060121. doi: E121 [pii].

2. Snipelisky D. Social media in medicine: A podium without boundaries. J Am Coll Cardiol. 2015;65(22):24592461. doi: 10.1016/j.jacc.2015.04.019 [doi].

3. Jamnadass $E$, Aboumarzouk $O$, Kallidonis $P$, et al. The role of social media and internet search engines in information provision and dissemination to patients with kidney stone disease: A systematic review from european association of urologists young academic urologists. J Endourol. 2018;32(8):673-684. doi: 10.1089/end.2018.0319 [doi].

4. Cawcutt K. Twitter me this-can social media revolutionize academic medicine? Infect Control Hosp Epidemiol. 2017;38(12):1501-1502. doi: 10.1017/ice.2017.242 [doi].

5. Choo EK, Ranney ML, Chan TM, et al. Twitter as a tool for communication and knowledge exchange in academic medicine: A guide for skeptics and novices. Med Teach. 2015;37(5):411-416. doi: 10.3109/0142159X.2014.993371 [doi].

6. Eysenbach G. Can tweets predict citations? metrics of social impact based on twitter and correlation with traditional metrics of scientific impact. J Med Internet Res. 2011;13(4):e123. doi: 10.2196/jmir.2012 [doi].

7. Rivas JG, Socarras MR, Patruno G, et al. Perceived role of social media in urologic knowledge acquisition among young urologists: A european survey. Eur Urol Focus. 2018;4(5):768-773. doi: S2405-4569(16)30168-7 [pii]. 
8. Topf JM, Sparks MA, Phelan PJ, et al. The evolution of the journal club: From osler to twitter. Am J Kidney Dis. 2017;69(6):827-836. doi: S0272-6386(17)30111-7 [pii].

9. Cosco TD. Medical journals, impact and social media: An ecological study of the twittersphere. CMAJ. 2015;187(18):1353-1357. doi: 10.1503/cmaj.150976 [doi].

10. Cardona-Grau D, Sorokin I, Leinwand G, Welliver C. Introducing the twitter impact factor: An objective measure of urology's academic impact on twitter. Eur Urol Focus. 2016;2(4):412-417. doi: S24054569(16)30008-6 [pii].

11. Nason GJ, O'Kelly F, Kelly ME, et al. The emerging use of twitter by urological journals. BJU Int. 2015;115(3):486-490. doi: 10.1111/bju.12840 [doi].

12. Loeb S, Bayne CE, Frey C, et al. Use of social media in urology: Data from the american urological association (AUA). BJU Int. 2014;113(6):993-998. doi: 10.1111/bju.12586 [doi].

13. Rivas JG, Socarras MR, Blanco LT. Social media in urology: Opportunities, applications, appropriate use and new horizons. Cent European J Urol. 2016;69(3):293-298. doi: 10.5173/ceju.2016.848 [doi].

14. Struck JP, Siegel F, Kramer MW, et al. Substantial utilization of facebook, twitter, YouTube, and instagram in the prostate cancer community. World J Urol. 2018;36(8):1241-1246. doi: 10.1007/s00345-018-2254-2 [doi].

15. Nolte AC, Nguyen KA, Perecman A, et al. Association between twitter reception at a national urology conference and future publication status. Eur Urol Focus. 2019. doi: S2405-4569(19)30140-3 [pii].

16. Loeb S. Social media makes global urology meetings truly global. : The influence of twitter. BJU Int. 2015;115(2):175. doi: 10.1111/bju.13027 [doi]. 
17. Loeb S, Taylor J, Butaney M, et al. Twitter-based prostate cancer journal club (\#ProstateJC) promotes multidisciplinary global scientific discussion and research dissemination. Eur Urol. 2019;75(5):881-882. doi: S0302-2838(19)30068-5 [pii].

18. Bayne CE, Cardona-Grau D, Hsieh MH. Introducing the pediatric urology journal club on twitter. J Pediatr Urol. 2017;13(1):2-3. doi: S1477-5131(17)30050-5 [pii].

19. Koo K, Shee K, Gormley EA. Following the crowd: Patterns of crowdsourcing on twitter among urologists. World J Urol. 2019;37(3):567-572. doi: 10.1007/s00345-018-2405-5 [doi].

20. Kind T. Professional guidelines for social media use: A starting point. AMA J Ethics. 2015;17(5):441-447. doi: 10.1001/journalofethics.2015.17.5.nlit1-1505 [doi].

21. Borgmann H, Cooperberg M, Murphy D, et al. Online professionalism-2018 update of european association of urology (@Uroweb) recommendations on the appropriate use of social media. Eur Urol. 2018;74(5):644650. doi: S0302-2838(18)30614-6 [pii].

22. Loeb S, Katz MS, Stork B. Update on the urology tag ontology: Standardized hashtags for social media in urology. Eur Urol. 2019. doi: S0302-2838(19)30418-X [pii].

23. Hayon S, Tripathi H, Stormont IM, Dunne MM, Naslund MJ, Siddiqui MM. Twitter mentions and academic citations in the urologic literature. Urology. 2019;123:28-33. doi: S0090-4295(18)31057-4 [pii].

24. Ciprut S, Curnyn C, Davuluri M, Sternberg K, Loeb S. Twitter activity associated with U.S. news and world report reputation scores for urology departments. Urology. 2017;108:11-16. doi: S0090-4295(17)30695-7 [pii]. 
25. Accreditation council for graduate medical education (ACGME). https://www.acgme.org/. Accessed March 1, 2019.

26. Canadian resident matching service (CaRMS). https://www.carms.ca/. Accessed March 1, 2019.

27. Hall N. The kardashian index: A measure of discrepant social media profile for scientists. Genome Biol. 2014;15(7):0. doi: 10.1186/s13059-014-0424-0 [doi].

28. Farber NJ, Koprowski CJ, Modi PK, et al. Twitter use among academic urology programs. Urology Practice. 2017;4(3):269-274. https://www.auajournals.org/doi/pdf/10.1016/j.urpr.2016.07.009.

29. Loeb S, Sengupta S, Butaney M, et al. Dissemination of misinformative and biased information about prostate cancer on YouTube. Eur Urol. 2019;75(4):564-567. doi: S0302-2838(18)30854-6 [pii]. 


\section{FIGURE(S) \& LEGENDS}

Figure 1: Twitter Utilization in Academic Urology

Figure 1A: Proportion of Urology Programs with Department-Specific Twitter Account

*Linear fitted response assessed by R2 statistic: 0.9336 (Good fit)

Figure 1B: Proportion of Academic Urologists with Twitter Account

Figure 2: Correlation of Academic Productivity with Twitter Utilization

Figure 2A: All Academic Urologists with Twitter Account (Log-10 scale, both axes) stratified by Hall Kardashian Index

Figure 2B: All Academic Urologists with Twitter Account (Log-10 scale, both axes) stratified by quadrant 